\title{
A New Series of 1,3,4-Oxadiazole Linked Quinolinyl-Pyrazole/Isoxazole Derivatives: Synthesis and Biological Activity Evaluation
}

\author{
V. Basavanna ${ }^{a}$, M. Chandramouli ${ }^{a}$, C. Kempaiah $^{b}$, U. K. Bhadraiah ${ }^{c}$ Chandra $^{d}$, \\ N. S. Lingegowda ${ }^{a}$, Shridevi Doddamani ${ }^{e}$, and S. Ningaiah ${ }^{a, *}$ \\ ${ }^{a}$ Department of Chemistry, Vidyavardhaka College of Engineering, Visvesvaraya Technological University, \\ Mysore, Karnataka, 570002 India \\ ${ }^{b}$ Department of Engineering Physics, HKBK College of Engineering, Bengaluru, Karnataka, 560045 India \\ ${ }^{c}$ Department of Chemistry, Yuvaraja's College, University of Mysore, Mysore, Karnataka, 570005 India \\ ${ }^{d}$ Department of Physics, The National Institute of Engineering (NIE), Mysore, Karnataka, 570008 India \\ ${ }^{e}$ Chemical Sciences and Technology Division, CSIR-NIIST, Thiruvananthapuram, Kerala, 695019 India \\ *e-mail: srijmn@vvce.ac.in
}

Received October 11, 2020; revised October 21, 2021; accepted November 8, 2021

\begin{abstract}
A series of 1,3,4-oxadiazole bridged pyrazole/isoxazole bearing quinoline derivatives has been designed and synthesized by a clean and convenient method. Structures of the newly synthesized compounds have been confirmed by FTIR, ${ }^{1} \mathrm{H}$ and ${ }^{13} \mathrm{C}$ NMR, and HRMS spectral data. The titled compounds have been evaluated for their molecular docking guided antimicrobial and anti-inflammatory activity. One of 1,3,4-oxadiazole bridged quinolinyl-pyrazole derivatives has interacted efficiently with E. Coli protein (PDB file: $1 \mathrm{KZN}$ ), and has been characterized by good antimicrobial activity against the majority of the tested pathogens. Another product has exhibited excellent anti-inflammatory activity.
\end{abstract}

Keywords: 1,3,4-oxadiazole, pyrazole, isoxazole, quinoline, molecular docking, antimicrobial, anti-inflammatory

DOI: $10.1134 / \mathrm{S} 1070363221110128$

\section{INTRODUCTION}

Quinoline and its derivatives are considered as versatile prodrugs, among those are antimalarial quinine (A) [1], chloroquine (B), and hydroxychloroquine (C)
(Fig. 1). The latter one is currently under clinical trials as a repurposed drug for COVID-19 [2].

Currently, many quinoline-containing drugs are available on the market $[3,4]$. Recently, three

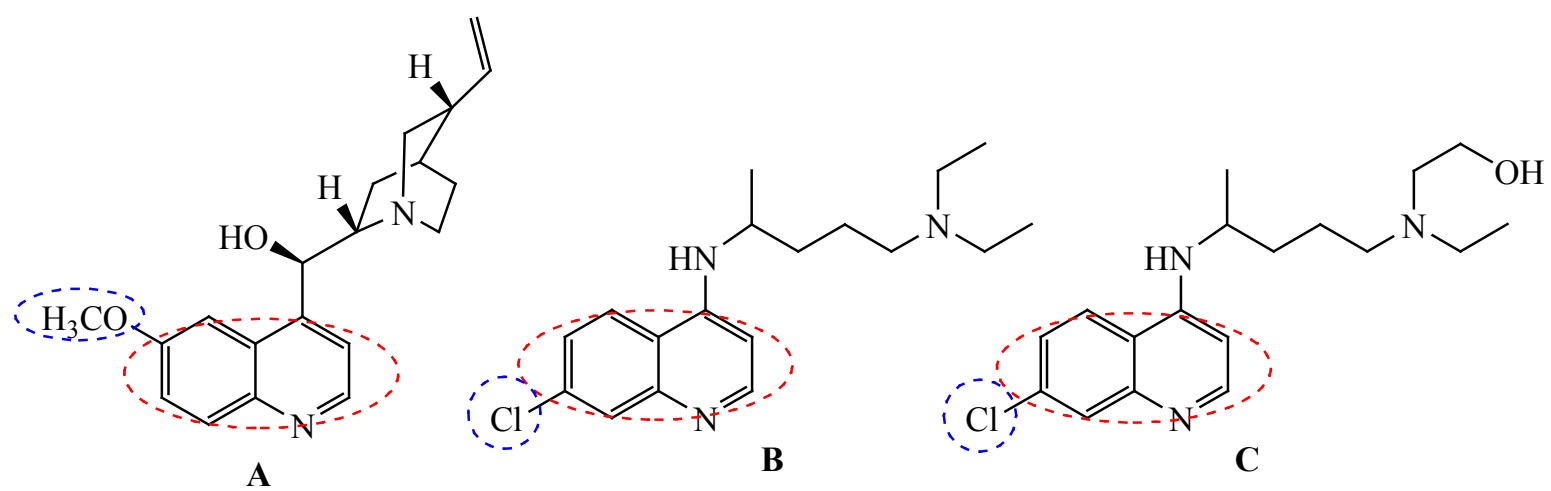

Fig. 1. Samples of promising quinoline alkaloids. 
Scheme 1. Synthesis of target compounds 11a-111.
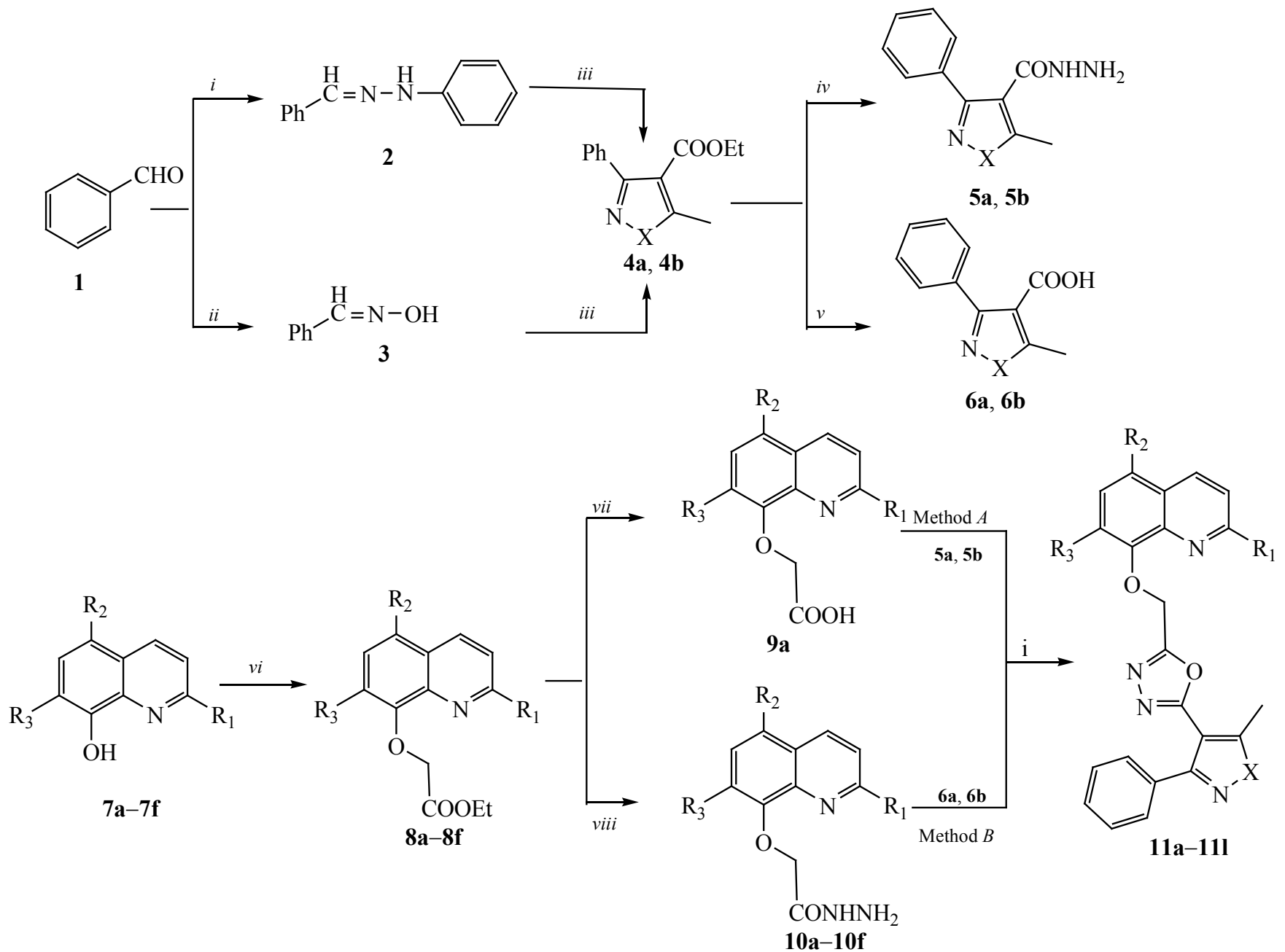

4a-6a $=X=\mathrm{N}-\mathrm{Ph}$, obtained from 2; $\mathbf{4 b}-\mathbf{6} \mathbf{b}=\mathrm{X}=\mathrm{O}$, obtained from 3; 9a and 10a: $\mathrm{R}_{1}=\mathrm{R}_{2}=\mathrm{R}_{3}=\mathrm{H}, \mathbf{1 0 b}: \mathrm{R}_{1}=\mathrm{H}, \mathrm{R}_{2}=\mathrm{Cl}$, $R_{3}=H, 10 c: R_{1}=H, R_{2}=R_{3}=C l, 10 d: R_{1}=M e, R_{2}=H, R_{3}=H, 10 e: R_{1}=M e, R_{2}=R_{3}=C l, 10 f: R_{1}=H, R_{2}=R_{3}=I, 11 a:$ $X=-, R_{1}=R_{2}=R_{3}=H, 11 b: X=-, R_{1}=H, R_{2}=C l, R_{3}=H, 11 c: X=-, R_{1}=H, R_{2}=R_{3}=C l, 11 d: X=-, R_{1}=M e, R_{2}=$ $R_{3}=H, 11$ e: $X=-, R_{1}=M e, R_{2}=R_{3}=C l, 11 f: X=-, R_{1}=H, R_{2}=R_{3}=I, 11 g: X=O, R_{1}=R_{2}=R_{3}=H, 11$ h: $X=O, R_{1}=H$, $\mathrm{R}_{2}=\mathrm{Cl}, \mathrm{R}_{3}=\mathrm{H}, 11 \mathrm{i}: \mathrm{X}=\mathrm{O}, \mathrm{R}_{1}=\mathrm{H}, \mathrm{R}_{2}=\mathrm{R}_{3}=\mathrm{Cl}, \mathbf{1 1} \mathrm{j}: \mathrm{X}=\mathrm{O}, \mathrm{R}_{1}=\mathrm{Me}, \mathrm{R}_{2}=\mathrm{R}_{3}=\mathrm{H}, \mathbf{1 1 k}: \mathrm{X}=\mathrm{O}, \mathrm{R}_{1}=\mathrm{Me}, \mathrm{R}_{2}=\mathrm{R}_{3}=\mathrm{Cl}, 11 \mathrm{l}$ : $\mathrm{X}=\mathrm{O}, \mathrm{R}_{1}=\mathrm{H}, \mathrm{R}_{2}=\mathrm{R}_{3}=\mathrm{I}$.

Reagents and conditions: (i) Phenyl hydrazine, ethanol, reflux, 3 h. (ii) Hydoxylamine hydrochloride, sodium acetate (1 eq.), ethanol, reflux, 12 h. (iii) Ethylacetoacetate, anhydrous zinc chloride (cat), reflux, 4 h, ethanol, (iv) Hydrazine hydrate, ethanol, reflux, $3 \mathrm{~h},(v)$ Sodium hydroxide, tetrahydo furan : water $(1: 1)$, reflux, $1 \mathrm{~h},(v i)$ Ethyl 2-chloroacetate, anhydrous potassium carbonate, dry acetone, reflux, $24 \mathrm{~h}$, (vii) $\mathrm{NaOH}$ (10\%), methanol, reflux, (viii) Hydrazine hydrate, ethanol, reflux, $1 \mathrm{~h}$. (ix) $\mathrm{POCl}_{3}$, $80^{\circ} \mathrm{C}$, Method $A: 24 \mathrm{~h}$ for the synthesis of $11 \mathrm{a}-11 \mathrm{c}$, Method $B: 10 \mathrm{~h}$ for the synthesis of 11a-111.

protein kinase inhibitors (Lenvatinib, Bosutinib, and Cabozantinib) and one Farnesyl transferase inhibitor (Tipifarnib) were presented as anticancer agents [5]. 1,3,4-Oxadiazole derivatives are well known for their antioxidant, anticancer, anticonvulsant, tyrosinase inhibitory, cathepsin K inhibitors, P-glycoprotein inhibitors, antimicrobial, antitumor, anti-inflammatory, and antiretroviral activities [6]. 
Table 1. Optimization of reaction conditions of synthesis of titled compounds

\begin{tabular}{c|c|c|c|c|c|c|c}
\hline \multirow{2}{*}{ Entry } & \multirow{2}{*}{ Compound } & \multicolumn{3}{|c|}{ Method A } & \multicolumn{3}{c}{ Method B } \\
\cline { 3 - 8 } & & time, $\mathrm{h}$ & weight, $\mathrm{g}$ & yield, \% & time, $\mathrm{h}$ & weight, g & yield, \% \\
\hline 1 & $\mathbf{1 1 a}$ & 24 & 0.37 & 79 & 10 & 0.40 & 87 \\
2 & $\mathbf{1 1 g}$ & 24 & 0.33 & 84 & 10 & 0.35 & 92 \\
\hline
\end{tabular}

Combination of quinoline and pyrazole structural blocks in molecules makes pharmacophores with respect to anti-cancer, interleukin, A3 adenosine receptor antagonists, anti-ulcer, and anti-inflammatory to be of certain potential [7]. Similarly, combination of quinoline and isoxazole heterocycles can result in formation of anti-TB [8,9], cytotoxic [10], antibacterial, antifungal [11], anti-inflammatory, and analgesic [12] compounds. Quinoline containing 1,3,4-oxadiazole derivatives exhibit pronounced biological activities $[13,14]$.

According to the earlier reports [15], the position 8 in quinoline was determined to be decisive for its biological activity. Generally, quinoline and its derivatives are considered as highly potent among derivatives of other five-membered heterocycles [16]. All the above inspired us to design and synthesize quinoline based compounds containing azoles combined by 1,3,4-oxadiazole bridge.

\section{RESULTS AND DISCUSSION}

The compounds $\mathbf{2}$ and $\mathbf{3}$ synthesized from benzaldehyde were subjected to cyclization reaction with ethyl acetoacetate in presence of the catalytic amount of anhydrous zinc chloride without using an additional solvent to give intermediates $\mathbf{4 a}$ and $\mathbf{4 b}$, respectively (Scheme 1).

In the following step, esters $\mathbf{4} \mathbf{a}, \mathbf{4} \mathbf{b}$ were treated with hydrazine hydrate in ethanol to afford $\mathbf{5 a}$ and $\mathbf{5 b}$, respectively. Subsequently, esters $\mathbf{4 a}, \mathbf{4 b}$ were hydrolyzed by $\mathrm{NaOH}(10 \%)$ in THF-water $(1: 1)$ medium to afford $\mathbf{6 a}$ and $\mathbf{6 b}$, respectively. Compounds $\mathbf{8 a}-\mathbf{8 f}$ were synthesized by treatment of a variety of 8 -hydroxyquinolines $7 \mathbf{a}-7 \mathbf{f}$ by ethyl 2-chloroacetate in acetone in presence of potassium carbonate. The isolated product $\mathbf{8 a}$ was subjected to hydrolysis by sodium hydroxide to afford acid $\mathbf{9 a}$. Refluxing of $\mathbf{8 a}-\mathbf{8 f}$ with hydrazine hydrate in ethanol led to the key intermediates $\mathbf{1 0 a}-\mathbf{1 0 f}$.

In the first approach to the target compounds, the compounds 11a and $11 \mathrm{~g}$ were synthesized by refluxing $9 \mathrm{a}$ with $\mathbf{5 a}$ or $\mathbf{5 b}$, respectively, in phosphorous oxychloride
(Scheme 1, method $A$ ). Alternatively, 10a was treated with $\mathbf{6 a}$ or $\mathbf{6 b}$ with formation of the target compounds 11a and 11g under the similar reaction conditions (Scheme 1, method $B$ ).

Optimization of reaction conditions for both methods is presented in Table 1 and favored method $B$. Probably poor solubility of $\mathbf{5 a}$ could lead to lower rate of the reaction. Accordingly, method $B$ was applied for synthesis of the other derivatives. It is noteworthy that higher yield was achieved in case of isoxazole ( 84 and 92\%) than pyrazole ( 79 and $87 \%$ ) due to absence of the bulky $\mathrm{N}$-phenyl group in the former one which minimized the partial positive charge on the carbonyl group.

Structures of the synthesized compounds were confirmed by FTIR, ${ }^{1} \mathrm{H}$ and ${ }^{13} \mathrm{C}$ NMR, and HRMS spectral data. Two singlets at $2.41 \mathrm{ppm}\left(\mathrm{CH}_{3}\right)$ and $5.35 \mathrm{ppm}\left(\mathrm{O}-\mathrm{CH}_{2}\right)$, and a doublet of doublets at 8.88$8.90 \mathrm{ppm}$ assigned to quinoline proton in ${ }^{1} \mathrm{H}$ NMR spectrum of compound 11a indicated its formation. In IR spectra, presence of characteristic bands at 1065$1089 \mathrm{~cm}^{-1}(\mathrm{~N}-\mathrm{N}), 1618-1643 \mathrm{~cm}^{-1}(\mathrm{C}=\mathrm{N})$, and $1229-1256 \mathrm{~cm}^{-1}(\mathrm{C}-\mathrm{O}-\mathrm{C})$ also supported formation of compounds 11a-111.

Biological activity. Molecular docking study. The molecular docking data are summarized in Table 2. The majority of target compounds demonstrated significant binding energy $(-11.57$ to $-7.97 \mathrm{~kJ} / \mathrm{mol})$ with the target sites possessing $\mathrm{H}$-bonds.

Antimicrobial activity. Results of antimicrobial activity (Table 3, Fig. 2) indicated that majority of products were of significant activity against most of the tested microbes. Compound 11d which contained the methyl group on quinoline ring was potent antimicrobial agent as well as 11a. The other compounds showed moderate activity. Compound $11 \mathbf{b}$ containing electron-withdrawing chlorine at $\mathrm{C}^{5}$ of quinoline displayed significant activity against gram-negative bacterial strain and demonstrated moderate activity against other pathogens. Compounds $\mathbf{1 1} \mathbf{c}$ and $\mathbf{1 1 i}$ that contained $\mathrm{Cl}$ at $\mathrm{C}^{5}$ and $\mathrm{C}^{7}$ on quinoline ring connected 
Table 2. Results of docking of compound 11a-11I with protein E. coli (PDB file: 1KZN)

\begin{tabular}{c|c|c|c|c|c|c|c}
\hline $\begin{array}{c}\text { Comp. } \\
\text { no. }\end{array}$ & $\begin{array}{c}\text { Binding energy, } \\
\mathrm{kJ} / \text { mol }\end{array}$ & $\begin{array}{c}\text { Ligand } \\
\text { efficiency }\end{array}$ & $\begin{array}{c}\text { Inhibition } \\
\text { constant }\end{array}$ & $\begin{array}{c}\text { vdW+H- } \\
\text { bond }+ \text { desolv } \\
\text { energy }\end{array}$ & $\begin{array}{c}\text { Number } \\
\text { of } \\
\text { H-bonds }\end{array}$ & Bonding residues & Bond length, A \\
\hline $\mathbf{1 1 a}$ & -10.92 & -0.38 & 9.94 & -12.39 & 1 & 1KZN:A:GLY77:HN & 2.050 \\
$\mathbf{1 1 b}$ & -7.97 & -0.27 & 1.44 & -9.51 & 1 & 1KZN:A:ASN46:HD21 & 1.793 \\
$\mathbf{1 1}$ & -10.27 & -0.33 & 29.60 & -11.85 & - & - & - \\
$\mathbf{1 1 d}$ & -11.57 & -0.39 & 3.30 & -13.07 & 1 & 1KZN:A:GLY77:HN & 2.148 \\
$\mathbf{1 1}$ & -10.35 & -0.32 & 25.69 & -11.93 & - & - & - \\
$\mathbf{1 1 f}$ & -9.41 & -0.30 & 126.89 & -10.85 & - & - & - \\
$\mathbf{1 1}$ & -8.96 & -0.26 & 268.80 & -10.78 & 1 & 1KZN:A:ASN46:HD21 & 1.746 \\
$\mathbf{1 1 h}$ & -10.29 & -0.29 & 28.60 & -12.08 & 1 & 1KZN:A:GLN135:HE21 & 2.234 \\
$\mathbf{1 1}$ & -8.65 & -0.23 & 453.37 & -10.42 & - & - & - \\
$\mathbf{1 1 j}$ & -10.79 & -0.30 & 12.40 & -12.62 & - & - & - \\
$\mathbf{1 1 k}$ & -10.16 & -0.27 & 35.42 & -11.95 & 1 & 1KZN:A:ASN46:HD21 & 1.812 \\
$\mathbf{1 1 1}$ & -8.90 & -0.24 & 298.46 & -10.71 & 1 & 1KZN:A:GLU50:OE1 & 2.710 \\
\hline
\end{tabular}

Table 3. Antimicrobial activity (MIC and MBC/MFC) of compounds 1,3,4-oxadiazole bridged quinolinyl-pyrazole/isoxazole analogues

\begin{tabular}{|c|c|c|c|c|c|c|c|c|c|c|c|c|c|c|}
\hline \multirow{4}{*}{ Compound } & \multicolumn{10}{|c|}{ Antibacterial activity } & \multirow{2}{*}{\multicolumn{4}{|c|}{ Antifungal activity }} \\
\hline & \multicolumn{4}{|c|}{ gram positive } & \multicolumn{6}{|c|}{ gram negative } & & & & \\
\hline & \multicolumn{2}{|c|}{ B. cereus } & \multicolumn{2}{|c|}{ S. aureus } & \multicolumn{2}{|c|}{ E. coli } & \multicolumn{2}{|c|}{ K. pneumonia } & \multicolumn{2}{|c|}{ S. flexneri } & \multicolumn{2}{|c|}{ C. albicans } & \multicolumn{2}{|c|}{ A. niger } \\
\hline & MIC & $\mathrm{MBC}$ & MIC & $\mathrm{MBC}$ & MIC & MBC & MIC & MBC & MIC & MBC & MIC & MFC & MIC & MFC \\
\hline $11 \mathrm{a}$ & 15 & 110 & 20 & 135 & 15 & 125 & 15 & 125 & 15 & 125 & 15 & 135 & 15 & 130 \\
\hline $11 b$ & 40 & 245 & 50 & 260 & 15 & 125 & 20 & 140 & 15 & 105 & 45 & 265 & 40 & 255 \\
\hline $11 \mathrm{c}$ & 30 & 195 & 40 & 200 & 10 & 100 & 10 & 105 & 40 & 245 & 40 & 250 & 45 & 270 \\
\hline 11d & 10 & 115 & 15 & 135 & 15 & 145 & 20 & 180 & 10 & 110 & 10 & 165 & 5 & 105 \\
\hline $11 \mathrm{e}$ & 20 & 140 & 30 & 175 & 25 & 160 & 15 & 130 & 30 & 180 & 25 & 145 & 20 & 140 \\
\hline $11 f$ & 50 & 245 & 30 & 180 & 50 & 250 & 40 & 230 & 35 & 195 & 20 & 150 & 15 & 215 \\
\hline $11 \mathrm{~g}$ & 25 & 150 & 30 & 170 & 20 & 140 & 15 & 110 & 20 & 130 & 15 & 130 & 20 & 145 \\
\hline $11 \mathrm{~h}$ & 40 & 235 & 55 & 280 & 20 & 130 & 10 & 105 & 15 & 120 & 55 & 255 & 55 & 275 \\
\hline $11 \mathrm{i}$ & 40 & 230 & 45 & 245 & 10 & 105 & 10 & 110 & 40 & 245 & 55 & 265 & 50 & 270 \\
\hline $11 \mathrm{j}$ & 20 & 135 & 30 & 160 & 25 & 140 & 30 & 150 & 25 & 140 & 15 & 125 & 20 & 140 \\
\hline $11 k$ & 35 & 190 & 40 & 230 & 30 & 180 & 45 & 235 & 35 & 210 & 25 & 180 & 15 & 135 \\
\hline 111 & 55 & 250 & 60 & 300 & 40 & 225 & 50 & 245 & 50 & 250 & 25 & 185 & 20 & 135 \\
\hline Chloramphenicol & 09 & 110 & 10 & 120 & 12 & 125 & 11 & 135 & 12 & 125 & & & - & - \\
\hline Fluconazole & & - & - & - & & - & & 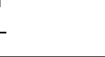 & & & & & 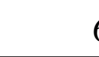 & 5 \\
\hline
\end{tabular}

by pyrazole/isoxazole bridge with 1,3,4-oxadiazole ring exhibited excellent activity against gram-negative bacterial strains (E. coli and K. pneumonia) at high concentration $(100 \mu \mathrm{g} / \mathrm{mL})$. So, compounds containing electron-withdrawing substituents demonstrated excellent activity against majority of the gram-negative bacteria, while compounds containing electron-donating groups displayed significant activity against both gram-positive and gram-negative bacterial strains. Compounds 11g and 11h were highly active against $S$. flexneri gram-negative 
Table 4. In vitro anti-inflammatory activity of 1,3,4-oxadiazole bridged quinolinyl-pyrazole/isoxazole analogues 11a-111

\begin{tabular}{l|c|c}
\hline Compound & Mean absorbance $\pm \mathrm{SD}^{\mathrm{a}}$ & Inhibition of denaturation, \% \\
\hline $\mathbf{1 1 a}$ & $0.2539 \pm 0.052$ & 24.82 \\
$\mathbf{1 1 b}$ & $0.3225 \pm 0.022$ & 58.55 \\
$\mathbf{1 1}$ & $0.3621 \pm 0.026$ & 78.02 \\
$\mathbf{1 1 d}$ & $0.2613 \pm 0.021$ & 28.46 \\
$\mathbf{1 1}$ & $0.2757 \pm 0.011$ & 35.54 \\
$\mathbf{1 1}$ & $0.2372 \pm 0.056$ & 16.61 \\
$\mathbf{1 1}$ & $0.2421 \pm 0.010$ & 19.02 \\
$\mathbf{1 1}$ & $0.3093 \pm 0.008$ & 52.06 \\
$\mathbf{1 1}$ & $0.3158 \pm 0.009$ & 55.26 \\
$\mathbf{1 1} \mathbf{1 1}$ & $0.2424 \pm 0.021$ & 19.17 \\
$\mathbf{1 1}$ & $0.2538 \pm 0.041$ & 24.77 \\
$\mathbf{1 1}$ & $0.2269 \pm 0.023$ & 11.15 \\
Diclofenac sodium & $0.3815 \pm 0.008$ & 87.56 \\
Control & $0.2034 \pm 0.016$ & - \\
\hline
\end{tabular}

${ }^{a} \mathrm{~S} . \mathrm{D}=$ standard deviation (average of three determinations).

bacteria but showed moderate activity against other tested strains which could be attributed to the presence of $\mathrm{Cl}$ on quinoline cycle in addition to isoxazole heterocycle. 1,3,4-Oxadiazole bridged pyrazolyl-quinoline compounds were more potent than isoxazolyl-quinoline compounds. The compounds containing iodine (11f, 111) exhibited the lowest antibacterial activity against all tested strains but showed good antifungal activity. Majority of the fungal strains were inhibited by compound 11a and 11d that contained the methyl group on quinoline ring while, other derivatives showed moderate antifungal activity.

Anti-inflammatory activity. Anti-inflammatory activity of the products were tested by inhibition of the albumin denaturation method according to Muzushima and Kabayashi with slight modification [17]. Diclofenac sodium was used as a standard (Table 4). The product 11c which contained $\mathrm{Cl}$ at $\mathrm{C}^{5}$ and $\mathrm{C}^{7}$ of quinoline ring demonstrated potent anti-inflammatory activity comparable with that of the standard drug. Compound 11b containing pyrazole and 1,3,4-oxadiazole and $\mathrm{Cl}$ at $\mathrm{C}^{5}$ demonstrated promising activity as well as $\mathbf{1 1 h}$ and 11i containing isoxazole ring. Compounds $\mathbf{1 1 f}$ and $\mathbf{1 1 l}$ carrying iodine at $\mathrm{C}^{5}$ and $\mathrm{C}^{7}$ of quinoline ring were of the lowest activity. According to the data summarized in Table 4, anti-inflammatory activity of products was influenced by electronegative $\mathrm{Cl}$ substituent and pyrazole ring attached to quinoline via 1,3,4-oxadiazole heterocycle.

\section{EXPERIMENTAL}

All reagents were purchased from Sigma Aldrich. Melting points were determined on a Buchi oil melting point apparatus and are uncorrected. IR spectra $(\mathrm{KBr}$ discs) were recorded on a SHIMADZU 8400 S FTIR

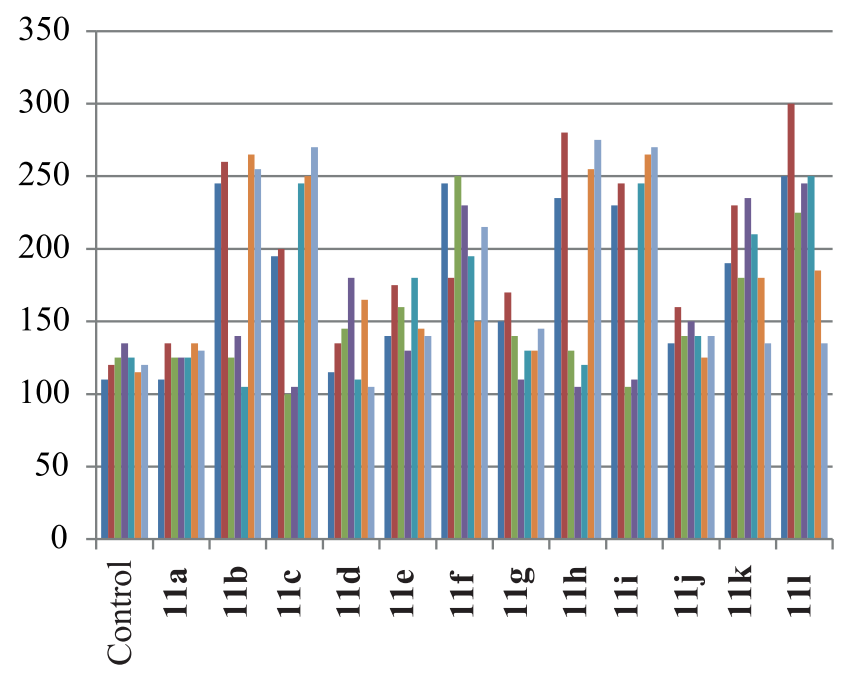

- Gram positive, B. cereus $\square$ Gram positive, S. aureus $\square$ Gram negative, E. coli $\square$ Gram negative, K. pneumonia n Gram negative, S. flexneri

- Antifungal activity, C. albicans

- Antifungal activity, A. niger

Fig. 2. Comparison of antimicrobial (MBC/MFC) activity of newly synthesized compounds $\mathbf{1 1 a}-\mathbf{1 1 1}$. 
spectrophotometer. ${ }^{1} \mathrm{H}$ and ${ }^{13} \mathrm{C}$ NMR spectra were measured on a Bruker Avance spectrometer $(400 \mathrm{MHz}$ for proton and $100 \mathrm{MHz}$ for carbon) respectively using $\mathrm{CDCl}_{3}$ as a solvent and TMS as an internal standard. High resolution mass spectra (HRMS) were measured by the direct infusion method on a MicroTOF-QII-ESI-QqTOF liquid chromatography mass spectrometer (Bruker Daltonics, Billerica, USA), electrospray ionization (ESI). Column chromatography was carried out using silica gel 60 (15-40 mm, Merck). TLC was performed on precoated (silica gel $60 \mathrm{~F} 254,0.2 \mathrm{~mm}$, Merck) plates and visualized under UV light and/or by iodine vapor.

Synthesis of intermediates 10a-10f. Intermediate compounds 10a-10f were synthesized by the reported earlier methods. 1-Benzylidene-2-phenylhydrazine (2) and benzaldehyde oxime (3) were synthesized from benzaldehyde [18, 19]. Ethyl 5-methyl-1,3-diphenyl1H-pyrazole-4-carboxylate (4a) and ethyl 5-methyl-3phenylisoxazole-4-carboxylate (4b) were synthesized by cyclization of compounds $\mathbf{2}$ and $\mathbf{3}$ with ethyl acetoacetate, respectively $[20,21]$. 5-Methyl-1,3-diphenyl-1 $H$ pyrazole-4-carbohydrazide (5a) and 5-methyl-3phenylisoxazole-4-carbohydrazide (5b) were synthesized by treating compounds $\mathbf{4 a}$ and $\mathbf{4 b}$ with hydrazine hydrate $[22,23]$. 5-Methyl-1,3-diphenyl- $1 H$-pyrazole4-carboxylic acid (6a) and 5-methyl-3-phenylisoxazole4-carboxylic acid (6b) were synthesized by hydrolysis in aqueous methanol [24, 25]. Ethyl 2-(quinolin-8-yloxy)acetates 8a-8f and 2-(quinolin-8-yloxy)acetohydrazides 10a-10f were synthesized by treating the corresponding 8-hydoxyquinoline $\mathbf{7 a - 7 f}$ with ethyl 2-chloroacetate followed by hydrazine hydrate [26, 27]. 2-(Quinolin-8yloxy)acetic acid 9a was obtained via hydrolysis of $\mathbf{8 a}$ in aqueous methanol.

Typical procedure for synthesis of 2-(5-methyl1,3-diphenyl-1H-pyrazol-4-yl)-5-[(quinolin-8-yloxy)methyl]-1,3,4-oxadiazole (11a) and 2-(5-methyl-3phenylisoxazol-4-yl)-5-[(quinolin-8-yloxy)methyl]1,3,4-oxadiazole (11g). Method A. 5-Methyl-1,3diphenyl-1H-pyrazole-4-carbohydrazide (5a, $0.29 \mathrm{~g}$, $1.00 \mathrm{mmol}$ ) and 5-methyl-3-phenylisoxazole-4carbohydrazide (5b, $0.21 \mathrm{~g}, 1.00 \mathrm{mmol})$ were placed in round bottom flasks fitted with a water cool condenser. To the flasks 2-(quinolin-8-yloxy)acetic acid (9a, $0.21 \mathrm{~g}$, $1.00 \mathrm{mmol}$ ) was added followed by $10 \mathrm{~mL}$ of phosphorous oxychloride.

Method B. 5-Methyl-1,3-diphenyl-1H-pyrazole-4carboxylic acid (6a, $0.27 \mathrm{~g}, 1.00 \mathrm{mmol})$ or 5-methyl- 3-phenylisoxazole-4-carboxylic acid $(6 \mathbf{b}, 0.21 \mathrm{~g}$, $1.00 \mathrm{mmol}$ ), 2-(quinolin-8-yloxy)acetohydrazide (10a, $0.22 \mathrm{~g}, 1.00 \mathrm{mmol}$ ) and $10 \mathrm{~mL}$ of phosphorous oxychloride were mixed together.

The other products were synthesized from the corresponding compounds following the method $B$.

The above reaction mixtures were refluxed on a water bath at $80^{\circ} \mathrm{C}$ upon monitoring by TLC, then reaction mixture was cooled down to $25^{\circ} \mathrm{C}$ and poured into crushed ice slowly with constant stirring. The resultant solution was neutralized by $20 \% \mathrm{KOH}$ and extracted by dichloromethane $(2 \times 25 \mathrm{~mL})$. The organic layer was washed with $5 \%$ hydrochloric acid $(2 \times$ $25 \mathrm{~mL}), 5 \%$ sodium hydroxide $(2 \times 25 \mathrm{~mL})$, brine solution $(1 \times 25 \mathrm{~mL})$, and finally with water $(25 \mathrm{~mL})$. The organic layer obtained was dried by anhydrous $\mathrm{Na}_{2} \mathrm{SO}_{4}$ and the solvent was removed under reduced pressure. The product was purified by column chromatography using hexane : ethyl acetate $(8: 2)$ as an eluent to afford the corresponding pure product as a white amorphous solid.

2-(5-Methyl-1,3-diphenyl-1 $\mathrm{H}$-pyrazol-4-yl)-5[(quinolin-8-yloxy)methyl]-1,3,4-oxadiazole (11a). IR spectrum, $v, \mathrm{~cm}^{-1}: 3261(\mathrm{C}-\mathrm{H}), 1622(\mathrm{C}=\mathrm{N}), 1241$ (Ar-O-C), $1068(\mathrm{~N}-\mathrm{N}) .{ }^{1} \mathrm{H}$ NMR spectrum, $\delta$, ppm: $2.41 \mathrm{~s}\left(3 \mathrm{H}, \mathrm{CH}_{3}\right), 5.35 \mathrm{~s}\left(2 \mathrm{H}, \mathrm{CH}_{2}\right), 7.40-7.60 \mathrm{~m}(14 \mathrm{H}$, ArH), 8.79-8.80 d (1H, ArH), 8.88-8.90 d. d (1H, ArH, $J=1.6,6.4 \mathrm{~Hz}) .{ }^{13} \mathrm{C}$ NMR spectrum, $\delta_{\mathrm{C}}, \mathrm{ppm}: 9.88\left(\mathrm{C}^{8}\right)$, $71.03\left(\mathrm{C}^{19}\right), 103.54\left(\mathrm{C}^{2}\right), 106.53\left(\mathrm{C}^{21}\right), 117.55\left(\mathrm{C}^{23}\right)$, $120.24\left(\mathrm{C}^{26}\right), 124.11\left(\mathrm{C}^{9}, \mathrm{C}^{13}\right), 126.80\left(\mathrm{C}^{11}\right), 127.25\left(\mathrm{C}^{22}\right)$, $127.59\left(\mathrm{C}^{14}, \mathrm{C}^{18}\right), 128.57\left(\mathrm{C}^{16}\right), 129.14\left(\mathrm{C}^{15}, \mathrm{C}^{17}\right), 129.52$ $\left(\mathrm{C}^{24}\right), 129.92\left(\mathrm{C}^{10}, \mathrm{C}^{12}\right), 132.82\left(\mathrm{C}^{7}\right), 135.13\left(\mathrm{C}^{25}\right), 137.72$ $\left(C^{3}\right), 139.34\left(C^{6}\right), 140.68\left(C^{28}\right), 147.48\left(C^{1}\right), 148.65$ $\left(C^{27}\right), 153.41\left(C^{20}\right), 163.40\left(C^{5}\right), 165.10\left(C^{4}\right)$. HRMS: $m / z: 460.1227 . \mathrm{C}_{28} \mathrm{H}_{21} \mathrm{~N}_{5} \mathrm{O}_{2}$.

2-\{[(5-Chloroquinolin-8-yl)oxy]methyl $\}-5-$ (5-methyl-1,3-diphenyl-1 H-pyrazol-4-yl)-1,3,4oxadiazole (11b). Yield 79\%. IR spectrum, $v, \mathrm{~cm}^{-1}$ : $3251(\mathrm{C}-\mathrm{H}), 1635(\mathrm{C}=\mathrm{N}), 1256(\mathrm{Ar}-\mathrm{O}-\mathrm{C}), 1064(\mathrm{~N}-\mathrm{N})$. ${ }^{1} \mathrm{H}$ NMR spectrum, $\delta$, ppm: $2.44 \mathrm{~s}\left(3 \mathrm{H}, \mathrm{CH}_{3}\right), 5.31 \mathrm{~s}$ $\left(2 \mathrm{H}, \mathrm{CH}_{2}\right), 7.39-7.59 \mathrm{~m}(13 \mathrm{H}, \mathrm{ArH}), 8.53-8.54 \mathrm{~d}(1 \mathrm{H}$, ArH), 8.98-8.99 d. d (1H, ArH, $J=1.2,5.2 \mathrm{~Hz}) .{ }^{13} \mathrm{C}$ NMR spectrum, $\delta_{\mathrm{C}}$, ppm: $9.90\left(\mathrm{C}^{8}\right), 71.32\left(\mathrm{C}^{19}\right), 103.33$ $\left(C^{2}\right), 106.83\left(C^{21}\right), 118.90\left(C^{23}\right), 122.37\left(C^{26}\right), 122.77$ $\left(\mathrm{C}^{9}, \mathrm{C}^{13}\right), 126.26\left(\mathrm{C}^{11}\right), 127.39\left(\mathrm{C}^{14}, \mathrm{C}^{18}\right), 127.82\left(\mathrm{C}^{22}\right)$, $128.41\left(\mathrm{C}^{16}\right), 129.02\left(\mathrm{C}^{15}, \mathrm{C}^{17}\right), 129.39\left(\mathrm{C}^{24}\right), 129.60$ $\left(\mathrm{C}^{10}, \mathrm{C}^{12}\right), 131.94\left(\mathrm{C}^{7}\right), 132.73\left(\mathrm{C}^{25}\right), 134.00\left(\mathrm{C}^{3}\right), 137.56$ $\left(\mathrm{C}^{6}\right), 139.79\left(\mathrm{C}^{28}\right), 147.41\left(\mathrm{C}^{1}\right), 152.72\left(\mathrm{C}^{27}\right), 154.16$ 
$\left(\mathrm{C}^{20}\right), 162.86\left(\mathrm{C}^{5}\right), 164.25\left(\mathrm{C}^{4}\right)$. HRMS: $m / z$ : 494.0671. $\mathrm{C}_{28} \mathrm{H}_{20} \mathrm{ClN}_{5} \mathrm{O}_{2}$.

2-\{[(5,7-Dichloroquinolin-8-yl)oxy]methyl $\}-5$ (5-methyl-1,3-diphenyl-1 H-pyrazol-4-yl)-1,3,4oxadiazole (11c). Yield $73 \%$. IR spectrum, $v, \mathrm{~cm}^{-1}: 3238$ $(\mathrm{C}-\mathrm{H}), 1630(\mathrm{C}=\mathrm{N}), 1251(\mathrm{Ar}-\mathrm{O}-\mathrm{C}), 1065(\mathrm{~N}-\mathrm{N}) .{ }^{1} \mathrm{H}$ NMR spectrum, $\delta$, ppm: $2.53 \mathrm{~s}\left(3 \mathrm{H}, \mathrm{CH}_{3}\right), 5.45 \mathrm{~s}(2 \mathrm{H}$, $\left.\mathrm{CH}_{2}\right), 7.41-7.61 \mathrm{~m}(12 \mathrm{H}, \mathrm{ArH}), 8.54-8.55 \mathrm{~d}(1 \mathrm{H}, \mathrm{ArH})$, 8.99-9.01 d. d (1H, ArH, $J=1.2,5.2 \mathrm{~Hz}) .{ }^{13} \mathrm{C}$ NMR spectrum, $\delta_{\mathrm{C}}$, ppm: $10.01\left(\mathrm{C}^{8}\right), 71.40\left(\mathrm{C}^{19}\right), 103.78$ $\left(\mathrm{C}^{2}\right), 107.11\left(\mathrm{C}^{21}\right), 120.85\left(\mathrm{C}^{23}\right), 122.48\left(\mathrm{C}^{26}\right), 122.88$ $\left(\mathrm{C}^{9}, \mathrm{C}^{13}\right), 126.37\left(\mathrm{C}^{11}\right), 127.47\left(\mathrm{C}^{14}, \mathrm{C}^{18}\right), 127.90\left(\mathrm{C}^{22}\right)$, $128.52\left(\mathrm{C}^{16}\right), 129.11\left(\mathrm{C}^{15}, \mathrm{C}^{17}\right), 129.54\left(\mathrm{C}^{24}\right), 129.72$ $\left(\mathrm{C}^{10}, \mathrm{C}^{12}\right), 132.04\left(\mathrm{C}^{7}\right), 132.92\left(\mathrm{C}^{25}\right), 134.24\left(\mathrm{C}^{3}\right), 137.73$ $\left(\mathrm{C}^{6}\right), 139.80\left(\mathrm{C}^{28}\right), 147.63\left(\mathrm{C}^{1}\right), 152.92\left(\mathrm{C}^{27}\right), 154.28$ $\left(\mathrm{C}^{20}\right), 162.90\left(\mathrm{C}^{5}\right), 164.45\left(\mathrm{C}^{4}\right)$. HRMS: $\mathrm{m} / z$ : 527.9884. $\mathrm{C}_{28} \mathrm{H}_{19} \mathrm{Cl}_{2} \mathrm{~N}_{5} \mathrm{O}_{2}$

2-(5-Methyl-1,3-diphenyl-1H-pyrazol-4-yl)-5-\{[(2-

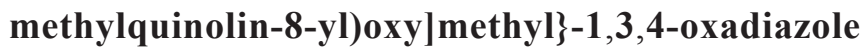
(11d). Yield 74\%. IR spectrum, $v, \mathrm{~cm}^{-1}: 3241(\mathrm{C}-\mathrm{H})$, $1628(\mathrm{C}=\mathrm{N}), 1242(\mathrm{Ar}-\mathrm{O}-\mathrm{C}), 1089(\mathrm{~N}-\mathrm{N}) .{ }^{1} \mathrm{H}$ NMR spectrum, $\delta$, ppm: $2.36 \mathrm{~s}\left(3 \mathrm{H}, \mathrm{CH}_{3}\right), 2.68 \mathrm{~s}\left(3 \mathrm{H}, \mathrm{CH}_{3}\right)$, $5.42 \mathrm{~s}\left(2 \mathrm{H}, \mathrm{CH}_{2}\right), 7.41-7.62 \mathrm{~m}(13 \mathrm{H}, \mathrm{ArH}), 8.79-8.80 \mathrm{~d}$ $(1 \mathrm{H}, \mathrm{ArH}), 8.87-8.88 \mathrm{~d}$. d $(1 \mathrm{H}, \mathrm{ArH}, J=1.6,6.4 \mathrm{~Hz}) .{ }^{13} \mathrm{C}$ NMR spectrum, $\delta_{\mathrm{C}}$, ppm: $9.96\left(\mathrm{C}^{8}\right), 25.32\left(\mathrm{C}^{29}\right), 71.12$ $\left(\mathrm{C}^{19}\right), 103.63\left(\mathrm{C}^{2}\right), 106.73\left(\mathrm{C}^{21}\right), 117.81\left(\mathrm{C}^{23}\right), 120.37$ $\left(\mathrm{C}^{26}\right), 124.31\left(\mathrm{C}^{9}, \mathrm{C}^{13}\right), 126.92\left(\mathrm{C}^{11}\right), 127.36\left(\mathrm{C}^{22}\right), 127.64$ $\left(\mathrm{C}^{14}, \mathrm{C}^{18}\right), 128.68\left(\mathrm{C}^{16}\right), 129.35\left(\mathrm{C}^{15}, \mathrm{C}^{17}\right), 129.74\left(\mathrm{C}^{24}\right)$, $130.04\left(\mathrm{C}^{10}, \mathrm{C}^{12}\right), 133.05\left(\mathrm{C}^{7}\right), 135.35\left(\mathrm{C}^{25}\right), 137.67\left(\mathrm{C}^{3}\right)$, $139.42\left(C^{6}\right), 140.71\left(C^{28}\right), 147.57\left(C^{1}\right), 148.81\left(C^{27}\right)$, $153.62\left(\mathrm{C}^{20}\right), 163.58\left(\mathrm{C}^{5}\right), 165.27\left(\mathrm{C}^{4}\right)$. HRMS: $m / z$ : 474.0633. $\mathrm{C}_{29} \mathrm{H}_{23} \mathrm{~N}_{5} \mathrm{O}_{2}$.

2-\{[(5,7-Dichloro-2-methylquinolin-8-yl)oxy]methyl \}-5-(5-methyl-1,3-diphenyl-1 $H$-pyrazol-4yl)-1,3,4-oxadiazole (11e). Yield 70\%. IR spectrum, $v$, $\mathrm{cm}^{-1}$ : $3252(\mathrm{C}-\mathrm{H}), 1623(\mathrm{C}=\mathrm{N}), 1233(\mathrm{Ar}-\mathrm{O}-\mathrm{C}), 1073$ $(\mathrm{N}-\mathrm{N}) .{ }^{1} \mathrm{H}$ NMR spectrum, $\delta$, ppm: $2.54 \mathrm{~s}\left(3 \mathrm{H}, \mathrm{CH}_{3}\right)$, $2.86 \mathrm{~s}\left(3 \mathrm{H}, \mathrm{CH}_{3}\right), 5.56 \mathrm{~s}\left(2 \mathrm{H}, \mathrm{CH}_{2}\right), 7.53-7.73 \mathrm{~m}(12 \mathrm{H}$, ArH), 8.55-8.56 d (1H, ArH), 8.99-9.00 d. d (1H, ArH, $J=1.2,5.2 \mathrm{~Hz}) .{ }^{13} \mathrm{C}$ NMR spectrum, $\delta_{\mathrm{C}}, \mathrm{ppm}: 10.11$ $\left(C^{8}\right), 24.52\left(C^{29}\right), 71.52\left(C^{19}\right), 103.69\left(C^{2}\right), 107.23\left(C^{21}\right)$, $120.79\left(\mathrm{C}^{23}\right), 122.46\left(\mathrm{C}^{26}\right), 123.08\left(\mathrm{C}^{9}, \mathrm{C}^{13}\right), 126.42\left(\mathrm{C}^{11}\right)$, $127.58\left(\mathrm{C}^{14}, \mathrm{C}^{18}\right), 127.83\left(\mathrm{C}^{22}\right), 128.63\left(\mathrm{C}^{16}\right), 129.24\left(\mathrm{C}^{15}\right.$, $\left.\mathrm{C}^{17}\right), 129.63\left(\mathrm{C}^{24}\right), 129.95\left(\mathrm{C}^{10}, \mathrm{C}^{12}\right), 132.13\left(\mathrm{C}^{7}\right), 132.78$ $\left(\mathrm{C}^{25}\right), 134.68\left(\mathrm{C}^{3}\right), 138.06\left(\mathrm{C}^{6}\right), 140.05\left(\mathrm{C}^{28}\right), 147.73\left(\mathrm{C}^{1}\right)$, $153.07\left(\mathrm{C}^{27}\right), 154.34\left(\mathrm{C}^{20}\right), 162.87\left(\mathrm{C}^{5}\right), 164.36\left(\mathrm{C}^{4}\right)$. HRMS: $m / z$ : $542.0048 . \mathrm{C}_{29} \mathrm{H}_{21} \mathrm{Cl}_{2} \mathrm{~N}_{5} \mathrm{O}_{2}$.
2-\{[(5,7-Diiodoquinolin-8-yl)oxy] methyl $\}-5-$ (5-methyl-1,3-diphenyl-1 $H$-py razol-4-yl)-1,3,4oxadiazole (11f). Yield $64 \%$. IR spectrum, $v, \mathrm{~cm}^{-1}: 3241$ $(\mathrm{C}-\mathrm{H}), 1634(\mathrm{C}=\mathrm{N}), 1245(\mathrm{Ar}-\mathrm{O}-\mathrm{C}), 1066(\mathrm{~N}-\mathrm{N}) .{ }^{1} \mathrm{H}$ NMR spectrum, $\delta$, ppm: $2.64 \mathrm{~s}\left(3 \mathrm{H}, \mathrm{CH}_{3}\right), 5.47 \mathrm{~s}(2 \mathrm{H}$, $\left.\mathrm{CH}_{2}\right), 7.55-7.75 \mathrm{~m}(12 \mathrm{H}, \mathrm{ArH}), 8.60-8.61 \mathrm{~d}(1 \mathrm{H}, \mathrm{ArH})$, 8.98-9.99 d. d (1H, ArH, $J=1.2,5.2 \mathrm{~Hz}) .{ }^{13} \mathrm{C}$ NMR spectrum, $\delta_{\mathrm{C}}$, ppm: $9.82\left(\mathrm{C}^{8}\right), 71.53\left(\mathrm{C}^{19}\right), 76.34\left(\mathrm{C}^{21}\right)$, $85.78\left(C^{23}\right), 102.54\left(C^{2}\right), 121.62\left(C^{26}\right), 123.11\left(C^{9}, C^{13}\right)$, $126.44\left(\mathrm{C}^{11}\right), 127.69\left(\mathrm{C}^{14}, \mathrm{C}^{18}\right), 128.79\left(\mathrm{C}^{22}\right), 128.78$ $\left(\mathrm{C}^{16}\right), 129.47\left(\mathrm{C}^{15}, \mathrm{C}^{17}\right), 129.68\left(\mathrm{C}^{24}\right), 129.93\left(\mathrm{C}^{10}\right.$, $\left.\mathrm{C}^{12}\right), 132.11\left(\mathrm{C}^{7}\right), 132.86\left(\mathrm{C}^{25}\right), 134.56\left(\mathrm{C}^{3}\right), 137.88$ $\left(C^{6}\right), 139.92\left(C^{28}\right), 147.77\left(C^{1}\right), 153.05\left(C^{27}\right), 154.35$ $\left(\mathrm{C}^{20}\right), 162.83\left(\mathrm{C}^{5}\right), 164.67\left(\mathrm{C}^{4}\right)$. HRMS: $m / z$ : 711.8631. $\mathrm{C}_{28} \mathrm{H}_{19} \mathrm{I}_{2} \mathrm{~N}_{5} \mathrm{O}_{2}$.

2-(5-Methyl-3-phenylisoxazol-4-yl)-5-[(quinolin-8yloxy)methyl]-1,3,4-oxadiazole (11g). IR spectrum, $v$, $\mathrm{cm}^{-1}$ : $3266(\mathrm{C}-\mathrm{H}), 1631(\mathrm{C}=\mathrm{N}), 1251(\mathrm{Ar}-\mathrm{O}-\mathrm{C}), 1069$ $(\mathrm{N}-\mathrm{N}) .{ }^{1} \mathrm{H}$ NMR spectrum, $\delta$, ppm: $2.40 \mathrm{~s}\left(3 \mathrm{H}, \mathrm{CH}_{3}\right)$, $5.13 \mathrm{~s}\left(2 \mathrm{H}, \mathrm{CH}_{2}\right), 7.45-7.55 \mathrm{~m}(9 \mathrm{H}, \mathrm{ArH}), 8.56-8.58 \mathrm{~d}$. d $(1 \mathrm{H}, \mathrm{ArH}, J=1.2,5.2 \mathrm{~Hz}), 8.96-8.97$ d. d $(1 \mathrm{H}, \mathrm{ArH}$, $J=1.6,5.6 \mathrm{~Hz}) .{ }^{13} \mathrm{C}$ NMR spectrum, $\delta_{\mathrm{C}}, \mathrm{ppm}: 14.81$ $\left(\mathrm{C}^{6}\right), 73.96\left(\mathrm{C}^{13}\right), 106.83\left(\mathrm{C}^{15}\right), 110.96\left(\mathrm{C}^{2}\right), 113.41\left(\mathrm{C}^{17}\right)$, $120.33\left(C^{20}\right), 124.64\left(C^{16}\right), 124.88\left(C^{8}, C^{12}\right), 128.46\left(C^{10}\right)$, $129.02\left(\mathrm{C}^{9}, \mathrm{C}^{11}\right), 129.62,\left(\mathrm{C}^{18}\right), 132.41\left(\mathrm{C}^{7}\right), 140.48\left(\mathrm{C}^{19}\right)$, $148.39\left(\mathrm{C}^{22}\right), 150.24\left(\mathrm{C}^{21}\right), 153.30\left(\mathrm{C}^{1}\right), 154.91\left(\mathrm{C}^{14}\right)$, $162.36\left(\mathrm{C}^{3}\right), 162.50\left(\mathrm{C}^{5}\right), 163.28\left(\mathrm{C}^{4}\right)$. HRMS: $m / z$ : 385.0467. $\mathrm{C}_{22} \mathrm{H}_{16} \mathrm{~N}_{4} \mathrm{O}_{3}$.

2-\{[(5-Chloroquinolin-8-yl)oxy] methyl $\}-5-(5-$ methyl-3-phenylisoxazol-4-yl)-1,3,4-oxadiazole (11h). Yield $87 \%$ yield. IR spectrum, $v, \mathrm{~cm}^{-1}: 3258(\mathrm{C}-\mathrm{H}), 1623$ $(\mathrm{C}=\mathrm{N}), 1243(\mathrm{Ar}-\mathrm{O}-\mathrm{C}), 1067(\mathrm{~N}-\mathrm{N}) .{ }^{1} \mathrm{H}$ NMR spectrum, $\delta$, ppm: $2.13 \mathrm{~s}\left(3 \mathrm{H}, \mathrm{CH}_{3}\right), 5.45 \mathrm{~s}\left(2 \mathrm{H}, \mathrm{CH}_{2}\right), 7.46-$ $7.59 \mathrm{~m}(8 \mathrm{H}, \mathrm{ArH}), 8.57-8.59$ d. d $(1 \mathrm{H}, \mathrm{ArH}, J=1.6$, $6.4 \mathrm{~Hz}), 9.00-9.01 \mathrm{~d} . \mathrm{d}(1 \mathrm{H}, \mathrm{ArH}, J=1.6,5.6 \mathrm{~Hz})$. ${ }^{13} \mathrm{C}$ NMR spectrum, $\delta_{\mathrm{C}}$, ppm: $13.72\left(\mathrm{C}^{6}\right), 72.57\left(\mathrm{C}^{13}\right)$, $106.38\left(\mathrm{C}^{15}\right), 110.38\left(\mathrm{C}^{2}\right), 118.46\left(\mathrm{C}^{17}\right), 122.34\left(\mathrm{C}^{20}\right)$, $126.16\left(\mathrm{C}^{16}\right), 127.61\left(\mathrm{C}^{8}, \mathrm{C}^{12}\right), 127.88\left(\mathrm{C}^{10}\right), 128.48\left(\mathrm{C}^{9}\right.$, $\left.\mathrm{C}^{11}\right), 128.72\left(\mathrm{C}^{18}\right), 131.75\left(\mathrm{C}^{7}\right), 132.87\left(\mathrm{C}^{19}\right), 139.84$ $\left(\mathrm{C}^{22}\right), 152.80\left(\mathrm{C}^{21}\right), 155.19\left(\mathrm{C}^{1}\right), 155.44\left(\mathrm{C}^{14}\right), 163.29$ $\left(C^{3}\right), 164.34\left(C^{5}\right), 165.59\left(C^{4}\right)$. HRMS: $m / z$ : 419.0205 . $\mathrm{C}_{22} \mathrm{H}_{15} \mathrm{ClN}_{4} \mathrm{O}_{3}$.

2-\{[(5,7-Dichloroquinolin-8-yl)oxy]methyl\}-5-(5methyl-3-phenylisoxazol-4-yl)-1,3,4-oxadiazole (11i). Yield 87\%. IR spectrum, $v, \mathrm{~cm}^{-1}: 3259(\mathrm{C}-\mathrm{H}), 1633$ $(\mathrm{C}=\mathrm{N}), 1238(\mathrm{Ar}-\mathrm{O}-\mathrm{C}), 1066(\mathrm{~N}-\mathrm{N}) .{ }^{1} \mathrm{H}$ NMR spectrum, $\delta$, ppm: $2.20 \mathrm{~s}\left(3 \mathrm{H}, \mathrm{CH}_{3}\right), 5.46 \mathrm{~s}\left(2 \mathrm{H}, \mathrm{CH}_{2}\right), 7.47-7.59 \mathrm{~m}$ (7H, ArH), 8.61-8.62 d. d (1H, ArH, $J=1.6,5.6 \mathrm{~Hz})$, 
9.03-9.05 d. d (1H, ArH, $J=1.6,5.6 \mathrm{~Hz}) .{ }^{13} \mathrm{C}$ NMR spectrum, $\delta_{\mathrm{C}}, \mathrm{ppm}: 14.29\left(\mathrm{C}^{6}\right), 73.07\left(\mathrm{C}^{13}\right), 110.38\left(\mathrm{C}^{15}\right)$, $110.44\left(\mathrm{C}^{2}\right), 119.57\left(\mathrm{C}^{17}\right), 122.54\left(\mathrm{C}^{20}\right), 127.16\left(\mathrm{C}^{16}\right)$, $127.83\left(\mathrm{C}^{8}, \mathrm{C}^{12}\right), 127.93\left(\mathrm{C}^{10}\right), 128.85\left(\mathrm{C}^{9}, \mathrm{C}^{11}\right), 128.93$ $\left(\mathrm{C}^{18}\right), 131.80\left(\mathrm{C}^{7}\right), 133.17\left(\mathrm{C}^{19}\right), 140.14\left(\mathrm{C}^{22}\right), 153.00$ $\left(\mathrm{C}^{21}\right), 155.34\left(\mathrm{C}^{1}\right), 155.53\left(\mathrm{C}^{14}\right), 164.03\left(\mathrm{C}^{3}\right), 164.54\left(\mathrm{C}^{5}\right)$, $166.12\left(\mathrm{C}^{4}\right)$. HRMS: $m / z$ : 452.5274. $\mathrm{C}_{22} \mathrm{H}_{14} \mathrm{Cl}_{2} \mathrm{~N}_{4} \mathrm{O}_{3}$.

2-(5-Methyl-3-phenylisoxazol-4-yl)-5-\{[(2methylquinolin-8-yl)oxy]methyl $\}$-1,3,4-oxadiazole (11j). Yield 84\%. IR spectrum, $v, \mathrm{~cm}^{-1}: 3251(\mathrm{C}-\mathrm{H})$, $1639(\mathrm{C}=\mathrm{N}), 1243(\mathrm{Ar}-\mathrm{O}-\mathrm{C}), 1085(\mathrm{~N}-\mathrm{N}) .{ }^{1} \mathrm{H}$ NMR spectrum, $\delta$, ppm: $2.22 \mathrm{~s}\left(3 \mathrm{H}, \mathrm{CH}_{3}\right), 2.53 \mathrm{~s}\left(3 \mathrm{H}, \mathrm{CH}_{3}\right)$, $5.51 \mathrm{~s}\left(2 \mathrm{H}, \mathrm{CH}_{2}\right), 7.16-7.17 \mathrm{~d}(1 \mathrm{H}, \mathrm{ArH}), 7.53-7.65 \mathrm{~m}$ (8H, ArH), 8.42-8.44 d. d (1H, ArH, $J=1.6,5.6 \mathrm{~Hz}) .{ }^{13} \mathrm{C}$ NMR spectrum, $\delta_{\mathrm{C}}, \mathrm{ppm}: 12.00\left(\mathrm{C}^{6}\right), 24.42\left(\mathrm{C}^{23}\right), 71.89$ $\left(\mathrm{C}^{13}\right), 107.12\left(\mathrm{C}^{15}\right), 111.13\left(\mathrm{C}^{2}\right), 113.76\left(\mathrm{C}^{17}\right), 120.13$ $\left(\mathrm{C}^{20}\right), 124.00\left(\mathrm{C}^{16}\right), 124.97\left(\mathrm{C}^{8}, \mathrm{C}^{12}\right), 128.56\left(\mathrm{C}^{10}\right), 128.89$ $\left(\mathrm{C}^{9}, \mathrm{C}^{11}\right), 132.76\left(\mathrm{C}^{18}\right), 136.57\left(\mathrm{C}^{7}\right), 140.63\left(\mathrm{C}^{19}\right), 148.68$ $\left(\mathrm{C}^{22}\right), 150.00\left(\mathrm{C}^{21}\right), 153.52\left(\mathrm{C}^{1}\right), 155.06\left(\mathrm{C}^{14}\right), 162.44$ $\left(C^{3}\right), 162.86\left(C^{5}\right), 163.47\left(C^{4}\right)$. HRMS: $m / z$ : 399.1336. $\mathrm{C}_{23} \mathrm{H}_{18} \mathrm{~N}_{4} \mathrm{O}_{3}$.

2-\{[(5,7-Dichloro-2-methylquinolin-8-yl)oxy]methyl\}-5-(5-methyl-3-phenylisoxazol-4-yl)-1,3,4oxadiazole (11k). Yield $82 \%$. IR spectrum, $v, \mathrm{~cm}^{-1}: 3263$ $(\mathrm{C}-\mathrm{H}), 1643(\mathrm{C}=\mathrm{N}), 1229(\mathrm{Ar}-\mathrm{O}-\mathrm{C}), 1077(\mathrm{~N}-\mathrm{N}) .{ }^{1} \mathrm{H}$ NMR spectrum, $\delta$, ppm: $2.23 \mathrm{~s}\left(3 \mathrm{H}, \mathrm{CH}_{3}\right), 2.45 \mathrm{~s}(3 \mathrm{H}$, $\left.\mathrm{CH}_{3}\right), 5.55 \mathrm{~s}\left(2 \mathrm{H}, \mathrm{CH}_{2}\right), 7.21-7.22 \mathrm{~d}(1 \mathrm{H}, \mathrm{ArH}), 7.46-$ $7.55 \mathrm{~m}(6 \mathrm{H}, \mathrm{ArH}), 8.61-8.63 \mathrm{~d} . \mathrm{d}(1 \mathrm{H}, \mathrm{ArH}, J=1.6$, $5.6 \mathrm{~Hz}) .{ }^{13} \mathrm{C}$ NMR spectrum, $\delta_{\mathrm{C}}$, ppm: $14.37\left(\mathrm{C}^{6}\right), 25.26$ $\left(\mathrm{C}^{23}\right), 73.12\left(\mathrm{C}^{13}\right), 110.47\left(\mathrm{C}^{15}\right), 111.14\left(\mathrm{C}^{2}\right), 118.76$ $\left(\mathrm{C}^{17}\right), 122.72\left(\mathrm{C}^{20}\right), 127.34\left(\mathrm{C}^{16}\right), 128.02\left(\mathrm{C}^{8}, \mathrm{C}^{12}\right), 128.01$ $\left(\mathrm{C}^{10}\right), 128.98\left(\mathrm{C}^{9}, \mathrm{C}^{11}\right), 128.79\left(\mathrm{C}^{18}\right), 131.91\left(\mathrm{C}^{7}\right), 133.27$ $\left(\mathrm{C}^{19}\right), 140.42\left(\mathrm{C}^{22}\right), 153.13\left(\mathrm{C}^{21}\right), 155.47\left(\mathrm{C}^{1}\right), 155.67$ $\left(\mathrm{C}^{14}\right), 164.42\left(\mathrm{C}^{3}\right), 164.97\left(\mathrm{C}^{5}\right), 166.32\left(\mathrm{C}^{4}\right)$. HRMS: $m / z$ : 466.8572. $\mathrm{C}_{23} \mathrm{H}_{16} \mathrm{Cl}_{2} \mathrm{~N}_{4} \mathrm{O}_{3}$.

2-\{[(5,7-Diiodoquinolin-8-yl)oxy]methyl\}-5-(5methyl-3-phenylisoxazol-4-yl)-1,3,4-oxadiazole (111). Yield 77\%. IR spectrum, $v, \mathrm{~cm}^{-1}: 3249(\mathrm{C}-\mathrm{H}), 1639$ $(\mathrm{C}=\mathrm{N}), 1243(\mathrm{Ar}-\mathrm{O}-\mathrm{C}), 1072(\mathrm{~N}-\mathrm{N}) .{ }^{1} \mathrm{H}$ NMR spectrum, $\delta$, ppm: $2.32 \mathrm{~s}\left(3 \mathrm{H}, \mathrm{CH}_{3}\right), 5.39 \mathrm{~s}\left(2 \mathrm{H}, \mathrm{CH}_{2}\right), 7.44-$ $7.56 \mathrm{~m}(7 \mathrm{H}, \mathrm{ArH}), 8.61-8.62$ d. d $(1 \mathrm{H}, \mathrm{ArH}, J=1.6$, $5.6 \mathrm{~Hz}), 8.89-8.91$ d. d $(1 \mathrm{H}, \mathrm{ArH}, J=1.6,5.2 \mathrm{~Hz}) .{ }^{13} \mathrm{C}$ NMR spectrum, $\delta_{\mathrm{C}}$, ppm: $13.81\left(\mathrm{C}^{6}\right), 73.23\left(\mathrm{C}^{13}\right), 76.97$ $\left(\mathrm{C}^{15}\right), 86.78\left(\mathrm{C}^{17}\right), 110.56\left(\mathrm{C}^{2}\right), 122.76\left(\mathrm{C}^{20}\right), 127.86\left(\mathrm{C}^{8}\right.$, $\left.\mathrm{C}^{12}\right), 127.26\left(\mathrm{C}^{10}\right), 128.90\left(\mathrm{C}^{9}, \mathrm{C}^{11}\right), 128.87\left(\mathrm{C}^{18}\right), 131.77$ $\left(\mathrm{C}^{7}\right), 133.47\left(\mathrm{C}^{19}\right), 140.38\left(\mathrm{C}^{22}\right), 147.42\left(\mathrm{C}^{16}\right), 153.15$ $\left(\mathrm{C}^{21}\right), 155.47\left(\mathrm{C}^{1}\right), 155.68\left(\mathrm{C}^{14}\right), 164.17\left(\mathrm{C}^{3}\right), 164.46$
$\left(\mathrm{C}^{5}\right), 166.23\left(\mathrm{C}^{4}\right)$. HRMS: $m / z$ : 636.2477. $\mathrm{C}_{22} \mathrm{H}_{14} \mathrm{I}_{2} \mathrm{~N}_{4} \mathrm{O}_{3}$ : 635.9155 .

Molecular docking study. Docking study was performed using Autodock-4.2. For evaluating the best binding sites the study was carried out for the crystal structure of E. coli (complex with Clorobiocin, PDB file $1 \mathrm{KZN}$ ) by the Lamarckian Genetic Algorithm computational method. To avoid interactions of solvent molecules, the software was calibrated by eliminating water and lower occupancy residues. Hydrogen bond with other docking constants represented the possible proximity of ligands with the receptor.

In vitro antibacterial and antifungal activity. The antimicrobial assay was performed by well-known methods [28]. The target compounds were tested for their antimicrobial activity against Gram-positive (Staphylococcus aureus (MTCC 96) and Bacillus cereus (MTCC 8372)) and Gram-negative (Escherichia coli (MTCC 724), Klebsiella pneumonia (MTCC 3384), and Shigella flexneri (MTCC 1457)) bacterial strains using nutrient agar medium by utilizing the paper disc diffusion method. Antifungal activity of synthesized compounds was tested on Candida albicans (MTCC 183) and Aspergillus niger (MTCC 281) fungal strains by the disc-diffusion method. Chloramphenicol and Fluconazole were used as standard drugs for antimicrobial and antifungal activity respectively. All experiments were done in triplicates and the results were represented in terms of diameter of the zone of inhibition. The MIC values were determined by the liquid dilution method against the same organisms, and absorbance of the test solutions was recorded on a spectrophotometer at $555 \mathrm{~nm}$.

In vitro anti-inflammatory activity. Antiinflammatory study was carried out by Protein denaturation by bovine serum albumin method [29]. The procedure described by Mamata D N and coworkers was used for preparing the solutions with slight modification [30]. The bovine serum albumin solution was obtained using tris buffer saline and $\mathrm{pH}$ adjusted to 6.8 by acetic acid. Series of test samples were prepared in $0.5 \mathrm{~mL}$ DMSO by treating different concentrations of target compounds with $0.5 \mathrm{~mL}$ of bovine serum albumin. A standard solution containing Diclofenac sodium $(0.05 \mathrm{~mL})$ and bovine serum albumin $(0.05 \mathrm{~mL})$ were used for comparison. The sample solutions were incubated at $37^{\circ} \mathrm{C}(20 \mathrm{~min})$ and then at $57^{\circ} \mathrm{C}(3 \mathrm{~min})$, then cooled down to room temperature and $2.5 \mathrm{~mL}$ of phosphate buffer were added. The mixtures were shaken thoroughly, and absorbance 
was measured on a UV-Vis spectrophotometer at $620 \mathrm{~nm}$. Protein denaturation in terms of percentage inhibition $(I, \%)$ of precipitation was calculate using the following formula:

$$
I=\left[V_{\mathrm{c}} / V_{\mathrm{s}}-1\right] \times 100,
$$

where $V_{\mathrm{c}}$-absorbance of control and $V_{\mathrm{s}}$-absorbance of sample solution.

\section{CONCLUSIONS}

The new 1,3,4-oxadiazole bridged quinoline linked pyrazole/isoxazole heterocyclic compounds have been synthesized. The molecular docking study of the final compounds against $E$. coli protein has indicated that those containing pyrazole heterocycle with methyl $\left(\mathrm{CH}_{3}\right)$ group at quinoline moiety have high binding energy and demonstrate excellent antimicrobial activity against the majority of the tested strains. Hence, the above templates can be considered as a useful lead for future development of potent drugs.

\section{ACKNOWLEDGMENTS}

The authors acknowledge the Management, Vidyavardhaka College of Engineering, Mysuru for the support to carry out research activity.

\section{CONFLICT OF INTEREST}

No conflict of interest was declared by the authors.

\section{SUPPLEMENTARY INFORMATION}

The online version contains supplementary material available at https://doi.org/10.1134/S1070363221110128.

\section{REFERENCES}

1. Singh, S.K., and Singh, S., Int. J. Pharm. Sci. Rev. Res., 2014, vol. 25, p. 295.

2. Awadhesh, K.S., Akriti, S., Altamash, S., Ritu, S., and Anoop, M., Diabetes Metab. Syndr., 2020, vol. 14 , p. 241-246.

https://doi.org/10.1016/j.dsx.2020.03.011

3. Arezoo, R.P., and Azar, T., Iran. J. Med Sci., 2017 , vol. 42, p. 115.

4. Ramadan, A.M., Mariam, A.A.S., Hanadi, Y.M., and Kamal, U.S., RSC Adv., 2020, vol. 10 p. 19867. https://doi.org/10.1039/D0RA02786C

5. Obaid, A., Suresh, K., and Rafi, M.H., Eur. J.
Med. Chem., 2015, vol. 97, p. 871.

https://doi.org/10.1016/j.ejmech.2014.07.044

6. Khalilullah, H., Ahsan, J.M., Hedaitullah, M., Khan, S., and Ahmed, B., Mini Rev. Med. Chem., 2012, vol. 12, p. 789.

https://doi.org/10.2174/138955712801264800

7. Basavanna, V., Ningaiah, S., Chandramouli, M., Sobha, A., and Doddamani, S., J. Iran. Chem. Soc., 2021, vol. 18, p. 1479.

https://doi.org/10.1007/s13738-020-02152-1

8. Annamaria, L., Jialin, M., Baojie, W., Yuehong, W., Scott, G.F., and Alan, P.K., J. Med. Chem., 2009, vol. 52, p. 2109.

https://doi.org/10.1021/jm900003c

9. Jialin, M., Hai, Y., Yuehong, W., Baojie, W., Dennis, P., Rong, H., and Scott, G.F., Bioorg. Med. Chem. Lett., 2010, vol. 20, p. 1263.

https://doi.org/10.1016/j.bmcl.2009.11.105

10. Sambasiva, R.P., Kurumurthy, C., Veeraswamy, B., Poornachandra, Y., Ganesh, K.C., and Narsaiah, B., Bioorg. Med. Chem. Lett., 2014, vol. 24, p. 1349.

https://doi.org/10.1016/j.bmcl.2014.01.038

11. Mariappan, B., Kasi, P., and Penugonda, R., Eur. J. Med. Chem., 2012, vol. 47, p. 608.

https://doi.org/10.1016/j.ejmech.2011.10.045

12. Rajanarendar, E., Nagi, R.M., Rama, K.S., Rama, M.K., Reddy, Y.N., and Rajam, M.V., Eur. J. Med. Chem., 2012, vol. 55, p. 273.

https://doi.org/10.1016/j.ejmech.2012.07.029

13. Rania, H., Samia, A.E., Noha, I.Z., Arwyn, T.J., and Andrew, D.W., Molecules., 2019, vol. 24, p. 1274.

https://doi.org/10.3390/molecules24071274

14. Sudeesh, K., and Gururaja, R., Organic Chem. Curr. Res., 2017, vol. 6, p. 1.

https://doi.org/10.4172/2161-0401.1000183

15. Damoder, R.M., Dilipkumar, U., and Blake, W.E., Chem. Sci., 2018, vol. 9, p. 1782.

https://doi.org/10.1039/C7SC04107A

16. Ibrahim, C., Ola, H.R., Tamer, M.I., Shery, S.H., ElSayeda, M.E.K., Aida, E.B., Ibrahim, M.E.A., and Hisham, A.N., Bioorg. Chem., 2018, vol. 78, p. 220. https://doi.org/10.1016/j.bioorg.2018.03.023

17. Muzushima, Y. and Kobayashi, M., J. Pharm. Pharmacol., 1968, vol. 20, p. 169.

https://doi.org/10.1111/j.2042-7158.1968.tb09718.x 
18. Mohammadizadeh, M.R. and Basti, F., J. Iran. Chem. Soc., 2015, vol. 12, p. 1171.

https://doi.org/10.1007/s13738-014-0578-4

19. Zhang, J.W., Yang, W.W., Chen, L.L., Chen, P., Wang, Y.B., and Chen, D.Y., Org. Biomol. Chem., 2019, vol. 17, p. 7461.

https://doi.org/10.1039/C9OB01387C

20. Katayama, H. and Oshiyama, T., Can. J. Chem., 1997, vol. 75, p. 913.

https://doi.org/10.1139/v97-109

21. Srikantamurthy, N., Vishalakshi, G.J., Jeyaseelan, S., Umesha, K.B., and Mahendra, M., Acta Cryst. E, 2013, vol. 69, p. 897.

https://doi.org/10.1107/S1600536813011410

22. Umesha, K.B., Rai, K.M., and Nayaka, M.H., Int. J. Biomed. Sci., 2009, vol. 4, p. 359-368.

23. Jagadish, S., Rajeev, N., Naveen Kumar, S.K., Kumar, K.S., Paul, M., Hegde, M., Sadashiva, M.P., Girish, K.S., and Rangappa, K.S., Mol. Cell. Biochem., 2016, vol. 414, p. 137.

https://doi.org/10.1007/s11010-016-2667-4
24. Shubakar, K., Umesha, K.B., Srikantamurthy, N., and Chethan, J., Bulg. Chem. Commun., 2013, vol. 45, p. 274.

25. Ningaiah, S., Bhadraiah, U.K., Doddaramappa, S.D., Keshavamurthy, S., and Javarasetty, C., Bioorg. Med. Chem., 2014, vol. 24, p. 245. https://doi.org/10.1016/j.bmcl.2013.11.029

26. Soliman, R. and Hammouda, N.A., J. Pharm. Sci., 1979, vol. 68, p. 1377. https://doi.org/10.1002/jps.2600681110

27. Naik, R.N., Pramod, H., Patil, S.C., Shridharamurthy, S., and Satyanarayana, S.B., Inventi Rapid: Med. Chem. 2013, vol. 2013, p. 29.

28. Khandelwal, N., Gautam, N., and Gautam, D.C., Int. J. Chem. Sci., 2013, vol. 125, p. 85. https://doi.org/10.1007/s12039-013-0363-4

29. Rahman, H., Eswaraiah, M.C., and Dutta, A.M., Am. Eur. J. Agri. Environ. Sci., 2015, vol. 15, p. 115. https://doi.org/10.5829/idosi.aejaes.2015.115.121

30. Naik, M.D., Bodke, Y.D., Prashantha, J., and Naik, J.K., Int. J. Chem. Sci., 2021, vol. 133, p. 1. https://doi.org/10.1007/s12039-020-01875-1 